\title{
Implementation of webcam-based hyperspectral imaging system
}

\section{Ali Balooch, Majid Nazeri, Hamed Abbasi}

Ali Balooch, Majid Nazeri, Hamed Abbasi, "Implementation of webcambased hyperspectral imaging system," Proc. SPIE 10539, Photonic Instrumentation Engineering V, 105391B (22 February 2018); doi: $10.1117 / 12.2290264$

SPIE. Event: SPIE OPTO, 2018, San Francisco, California, United States 


\title{
Implementation of webcam-based hyperspectral imaging system
}

\author{
Ali Balooch ${ }^{\mathrm{a}}$, Majid Nazeri*a ${ }^{*}$,Hamed Abbasi ${ }^{\dagger \mathrm{b}}$ \\ ${ }^{a}$ THz and Laser Spectroscopy Lab., Department of Photonics, Faculty of Physics, University of \\ Kashan, 87317-53153 Kashan, Iran \\ ${ }^{\mathrm{b}}$ Biomedical Laser and Optics Group, Department of Biomedical Engineering, University of Basel, \\ Gewerbestrasse 14, CH-4123 Allschwil, Switzerland
}

\begin{abstract}
In the present work, a hyperspectral imaging system (imaging spectrometer) using a commercial webcam has been designed and developed. This system was able to capture two-dimensional spectra (in emission, transmission and reflection modes) directly from the scene in the desired wavelengths. Imaging of the object is done directly by linear sweep (pushbroom method). To do so, the spectrometer is equipped with a suitable collecting lens and a linear travel stage. A 1920 x 1080 pixel CMOS webcam was used as a detector. The spectrometer has been calibrated by the reference spectral lines of standard lamps. The spectral resolution of this system was about $2 \mathrm{~nm}$ and its spatial resolution was about $1 \mathrm{~mm}$ for a $10 \mathrm{~cm}$ long object. The hardware solution is based on data acquisition working on the USB platform and controlled by a LabVIEW program. In this system, the initial output was a three-dimensional matrix in which two dimensions of the matrix were related to the spatial information of the object and the third dimension was the spectrum of any point of the object. Finally, the images in different wavelengths were created by reforming the data of the matrix. The free spectral range (FSR) of the system was 400 to $1100 \mathrm{~nm}$. The system was successfully tested for some applications, such as plasma diagnosis as well as applications in food and agriculture sciences.
\end{abstract}

Keywords: Hyperspectral imaging, spectroscopy, pushbroom method, CMOS

\section{INTRODUCTION}

Hyperspectral imaging a is a novel technique to obtain both spectral and spatial information of an object. They could work in transmission, reflection or emission modes. Over the past several years, hyperspectral imaging becomes one the fastest-growing tools for non-destructive analyses in different fields [1-10]. Basically the applications of it are very similar to the applications of normal spectroscopy, but it can provide spectrum associated with the spatial information. Therefore, in comparison to conventional black and white or colorful photography, they are capable of providing information related to the chemical composition of the objects. Moreover, there has been a remarkable increase in the number of remote sensing application of hyperspectral imaging systems [11-13]. It could be used for non-destructive quality assessment of food and agricultural products [14-17], since nowadays the food and agriculture and industries are concerned about the high quality and sustainable production and delivery of consistent and safe food products to an increasing extent $[18,19]$. This device could also connect to fluorescence spectroscopy system for better classification [20-22]. In addition, they could work with a large variety of light sources in ultraviolet, visible and near-infrared regimes. Similar to normal spectrometers, hyperspectral imaging systems could be used for studying the emission profile of the plasma sources related with different elements or to determine plasma temperature/electron density/electric filed profile $[23,24]$. A post-processing data analysis is required (either unsupervised or supervised) to bring more power to the initial output of the systems, both qualitatively and quantitatively, they range from simple statistical classification/discrimination methods to advanced neural network-based image processing techniques for multivariate image analysis [25-29]. Prior to the applied classification method (either linear or nonlinear) a pre-data reduction method could help to improve the accuracy of the method. This study intends to examine the capability of the low-cost hyperspectral system (using a consumer-grade webcam instead of the scientific camera) for different applications in both reflection and emission modes, since reducing the final price of such instruments could bring them in a wide range of applications.

*m_nazeri@kashanu.ac.ir; phone +98 (0)31 559130 58; http://mnazeri.faculty.kashanu.ac.ir

thamed.abbasi@unibas.ch; phone +41 (0)61 20754 61; http://dbe.unibas.ch/blog

Photonic Instrumentation Engineering V, edited by Yakov G. Soskind,

Craig Olson, Proc. of SPIE Vol. 10539, 105391B - (c) 2018 SPIE

CCC code: $0277-786 X / 18 / \$ 18 \cdot$ doi: $10.1117 / 12.2290264$

Proc. of SPIE Vol. 10539 105391B-1 


\section{MATERIALS AND METHODS}

\subsection{Optical design}

There are several approaches to develop hyperspectral imaging systems including Whiskbroom, Pushbroom, Staring and Snapshot-based methods. Table 1 shows specifications, benefits, and limitations of the mentioned methods. Pushbroom method, which works based on a linear scanning, was used in this study.

Table 1. Specifications, benefits, and limitations of the methods for developing hyperspectral imaging systems.

\begin{tabular}{lllll}
\hline & Whiskbroom & Pushbroom & Staring & Snapshot \\
\hline Scanning & 2D & 1D & Spectral scan & No scan \\
\hline Detector & 1D & 2D & 2D & 2D \\
\hline Dispersion & Prism/ Grating & Prism/ Grating & Filter/ Interferometer & Prism/ Grating \\
\hline Free spectral range & Wide & Wide & Mediate & Wide \\
\hline Spectral resolution & High & High & Mediate & Mediate \\
\hline Spatial resolution & High & High & High & Very low \\
\hline Collection time & Very long & Long & Mediate & Fast \\
\hline Optical density & High & High & Low & High \\
\hline
\end{tabular}

A typical pushboom-based hyperspectral imaging system consists of an imaging spectrometer and a linear translation stage. The imaging spectrometer is a normal spectrometer which a convex lens is added in front of its entrance slit. Figure 1 shows the schematic of the developed imaging spectrometer.

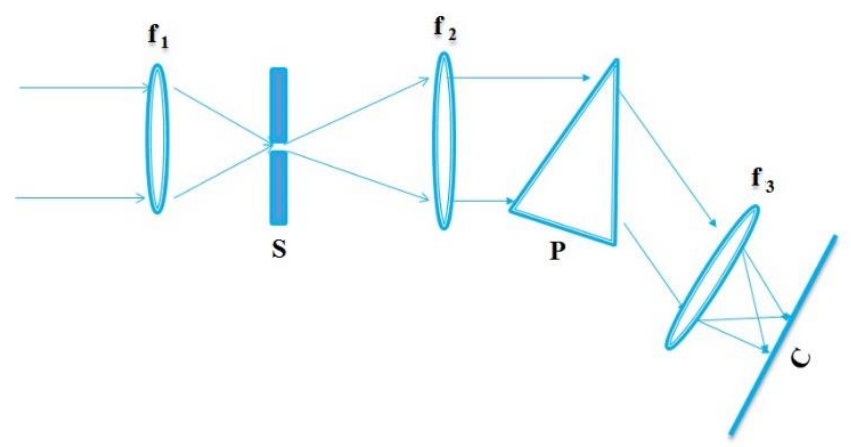

Figure 1. Schematic of the optical design of the imaging spectrometer: $f_{1}, f_{2}$, and $f_{3}$ stand for lens 1 , lens 2 and lens 3 with the focal lengths of 50, 75 and $75 \mathrm{~mm}$, respectively. Also, S, P, and C stand for the slit, dispersive prism, and camera (webcam), respectively.

In this study, a prism-based spectrometer controlled by LabVIEW was developed in order to acquire the spectra in the interval of 400 to $1100 \mathrm{~nm}$. The collection lens $\left(f_{1}\right)$ collect and send the light to the entrance slit of the spectrometer. The entrance light illuminates the slit $(\mathrm{S})$, which was placed in the focal plane of the collimator lens $\left(\mathrm{f}_{2}\right)$. The parallel light, behind the collimator lens, passes through the dispersion optic (a triangle flint prism in this study (P)), where it is diffracted depending on the wavelength $\lambda$. The prism was installed at the minimum angle of deviation to have optimum performance. In minimum deviation angle, the wavelength of interest travel parallel to the base of the prism, and the angle of incidence is equal to the angle of refraction [30, 31]. The imaging lens ( $\left.f_{3}\right)$ forms a wavelength-dependent image 
of the entrance slit on the detector (C). A 1920 x 1080 pixel CMOS webcam (8-bit) was used as a detector. Figure 1 shows a picture of the used webcam.

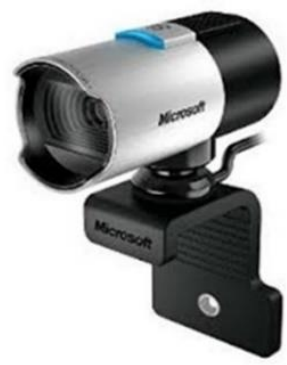

Figure 2. The picture of the webcam with CMOS sensor used as a detector.

The imaging spectrometer was calibrated using the atomic emission lines os the sodium $(\mathrm{Na})$ and mercury $(\mathrm{Hg})$ lamps [32]. Figure 3 shows the external view of the developed imaging spectrometer.

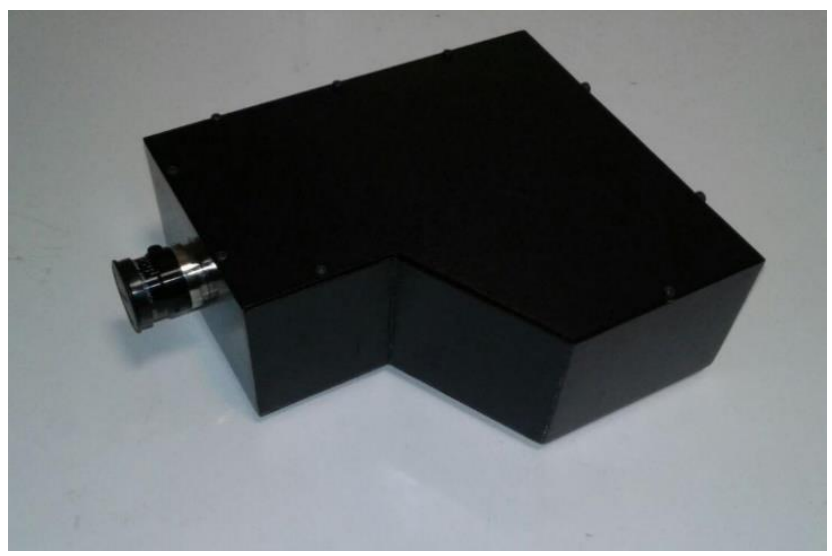

Figure 3. The external view of the developed imaging speedometer.

\subsection{Motorized translation stage}

A motorized translation stage which could provide electronically controlled linear motion along one dimension is necessary for pushbroom-based hyperspectral imaging systems to scan the entire sample. A motorized translation stage with low backlash and variable speed was developed to move either the imaging spectrometer or the sample. The load capacity was enough to hold imaging spectrometer without changing the speed. The maximum travel range of the developed translation stage was $20 \mathrm{~cm}$. The LabVIEW program was used to control the motor. Figure 4 shows an image of the motorized stage on the table.

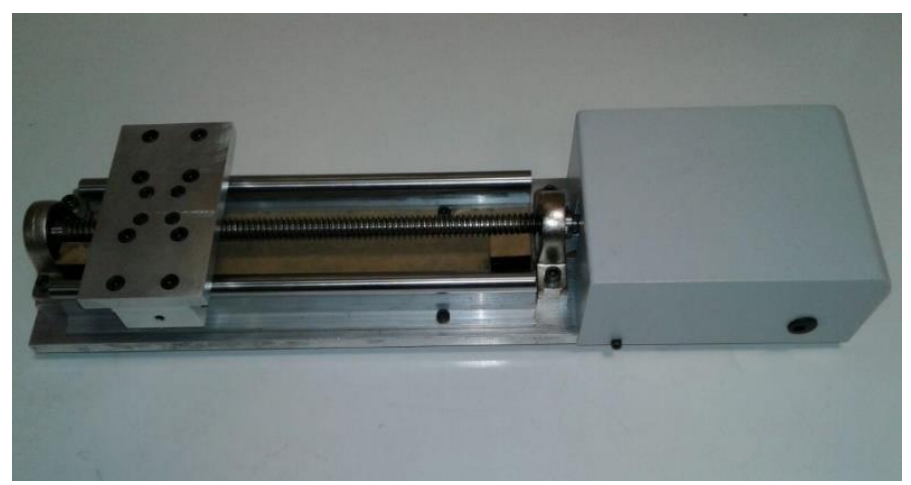

Figure 4. The image of the developed translation stage. 


\subsection{Software}

A LabVIEW code was written to process the initial data from the CMOS sensor and reconstruct the image at the desired wavelength. The code was capable of exporting the data in Excel file format or the reconstructed image in BMP format. Figure 5 shows a screenshot of the user interface of the running program on the PC.

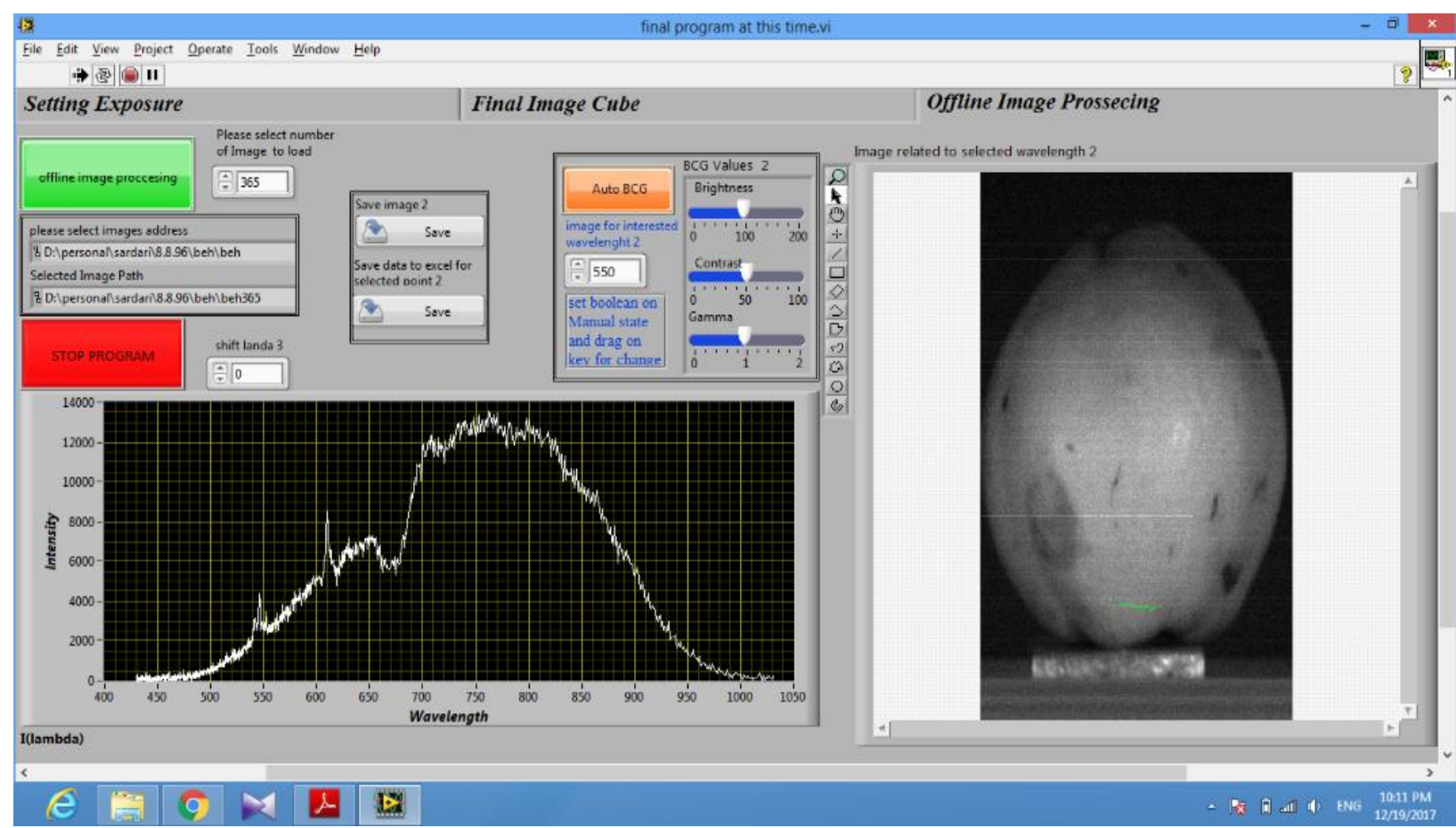

Figure 5. Screenshot of the user interface of the developed LabVIEW program. A spectrum of a single point of the sample and a reconstructed image of the entire sample are located in the lower left corner and right part of the screen, respectively.

\section{RESULTS}

\subsection{Reflection and transmission mode}

In the reflection and transmission modes of working, an external broadband light source is necessary to illuminate the samples. In the current study, a halogen lamp was used a light source. Figure 6 shows the reconstructed spectral images of the quince fruit collected in 3 different wavelengths $(550,700$ and $900 \mathrm{~nm})$ in reflection mode.
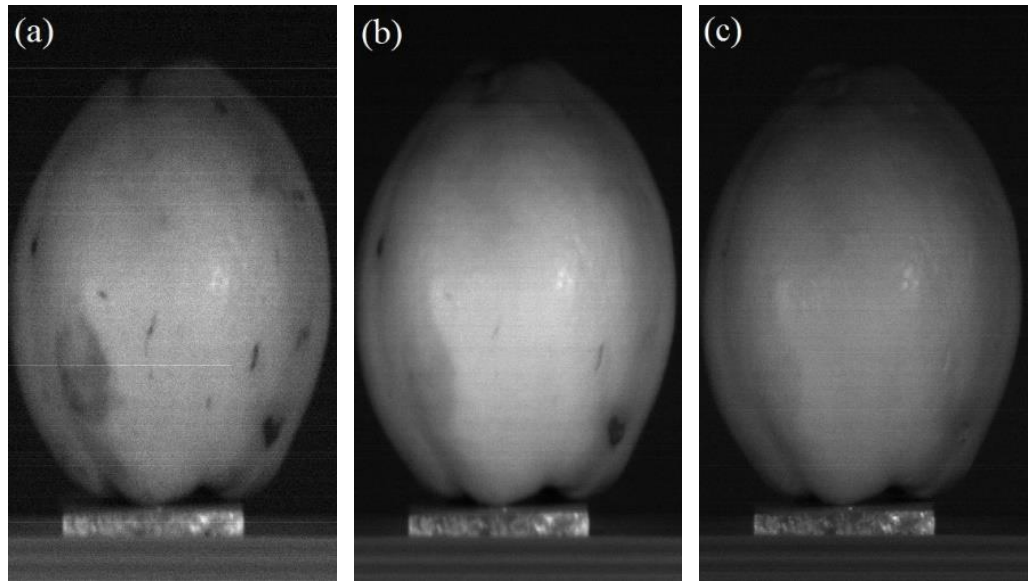

Figure 6. Reconstructed image of the quince fruit in (a) $550 \mathrm{~nm}$, (b) $700 \mathrm{~nm}$, and (c) $900 \mathrm{~nm}$. Defects are more clear in shorter wavelengths. 
As it is clear from the pictures, some defect of the fruit is visible in the $550 \mathrm{~nm}$, while it is not visible in $900 \mathrm{~nm}$. Figure 7 shows the reconstructed spectral images (in reflection mode) of a frozen orange fruit collected in 6 different wavelengths in visible and near-infrared (from 550 to $900 \mathrm{~nm}$ ).
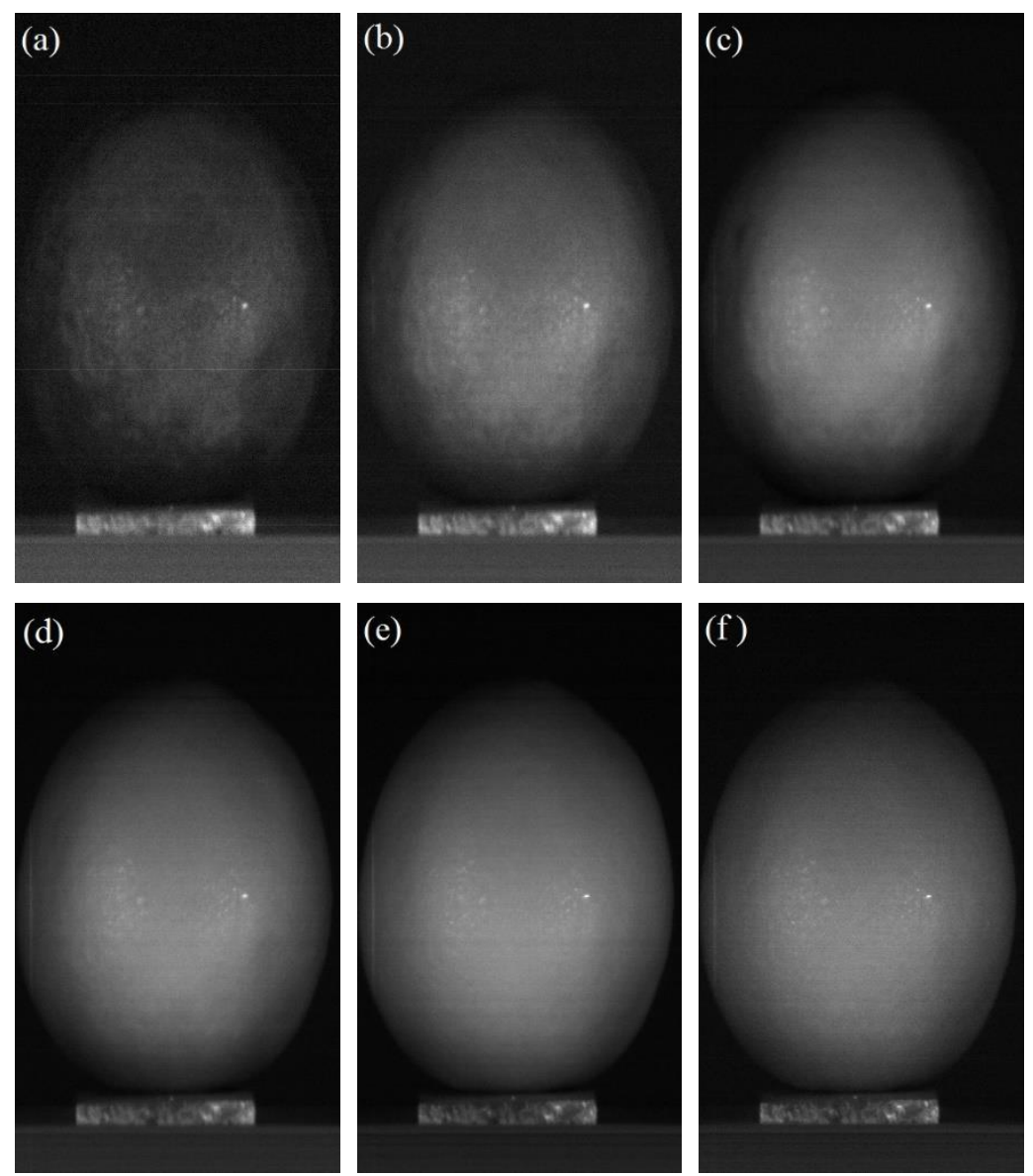

Figure 7. Reconstructed image of a frozen orange fruit in (a) $550 \mathrm{~nm}$, (b) $600 \mathrm{~nm}$, (c) $650 \mathrm{~nm}$, (d) $700 \mathrm{~nm}$, (e) $800 \mathrm{~nm}$, and (f) $900 \mathrm{~nm}$.

Figures 8 and 9 show the spectral images of some leaves in the different wavelengths (compared to the normal RGB image).
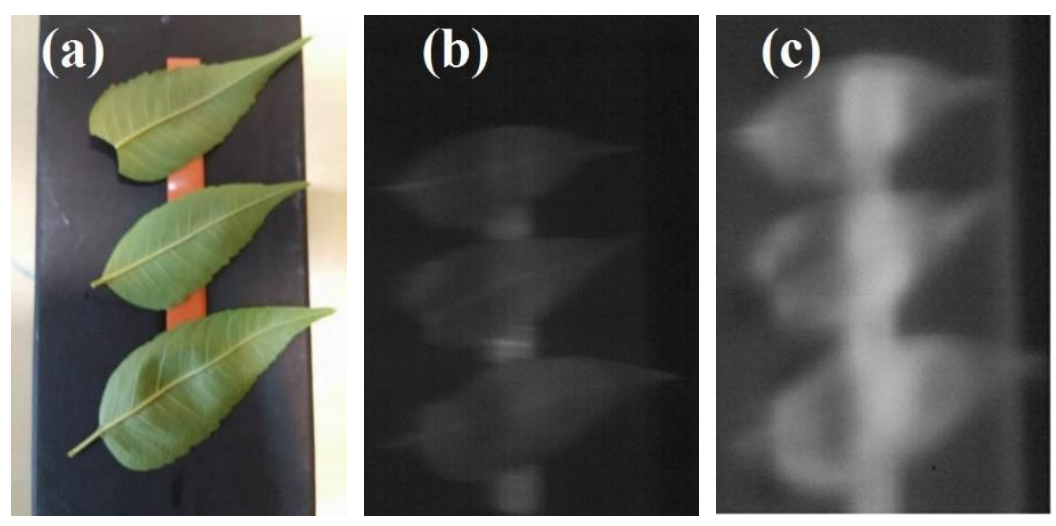

Figure 8. (a) Normal RGB image of the leaf, (b) $593 \mathrm{~nm}$ image of the leaf, and (c) $819 \mathrm{~nm}$ image of the leaf. An orange adhesive tape behind the leaf is visible in the $819 \mathrm{~nm}$ image, while is not visible in the normal RGB image. 

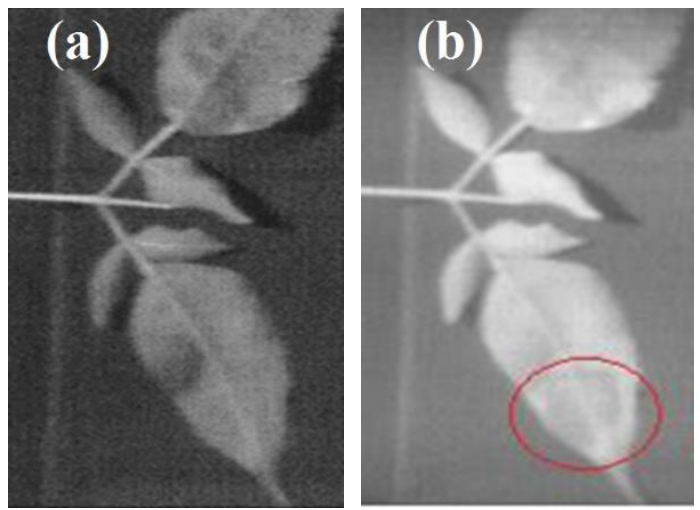

Figure 9. (a) $593 \mathrm{~nm}$ image of the leaf, and (b) $819 \mathrm{~nm}$ image of the leaf. A liquid glue behind the leaf in visible in the lower

right corner of the $819 \mathrm{~nm}$ image.

As it is clear from both figures 8 and 9, due to low absorption of the leaf in $819 \mathrm{~nm}$, the reflected light (at this wavelength) from the glue/adhesive tape behind the leaf can pass the leaf.

\subsection{Emission mode}

Unlike transmission and reflection modes, the hyperspectral imaging system could work stand-alone (no need for external light source), since it collects the light directly emitted from the object of study. Figure 10 shows the reconstructed spectral images of an atmospheric pressure cold plasma jet in emission mode.
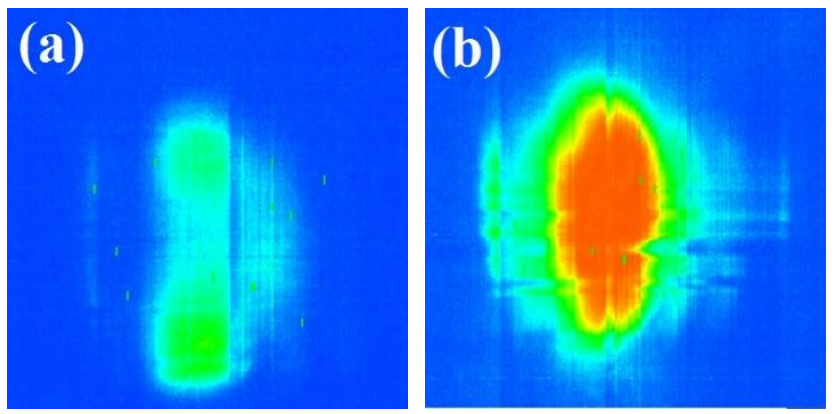

Figure 10. (a) $770.5 \mathrm{~nm}$ image of the plasma jet, and (b) $777.5 \mathrm{~nm}$ image of the plasma jet. The emission profile of ionized oxygen is visible in the 777.5 image.

\section{DISCUSSION}

The results showed that the developed system could successfully reconstruct the spectral images in different wavelength in visible and near-infrared regions with good spectral and spatial resolutions. The spectral resolution of this system was about $2 \mathrm{~nm}$ and its spatial resolution was about $1 \mathrm{~mm}$ for a $10 \mathrm{~cm}$ long object. Having less spatial resolution in the reconstructed images of the plasma jet in the emission mode is due to fluctuating of the plasma flame. On the other hand, due to the stability of the samples, in both transmission and reflection modes, the spectral resolution was better. In order to capture spectral images with a high spatial resolution from fractionating sources, faster methods like staring or snapshot could be used.

\section{CONCLUSION}

A webcam-based hyperspectral imaging system (imaging spectrometer) controlled by LabVIEW software has been designed and implemented. The hyperspectral imaging system was able to capture two-dimensional emission/reflection spectra directly from the scene in the desired wavelengths. The spectrometer was a fiber-free prism-based spectrometer; imaging of the object was done directly by linear sweep (pushbroom method). The spectrometer was equipped with a suitable collecting lens and a linear travel stage, to do so. A CMOS webcam was used as a detector. Finally, the system 
was employed in both reflection and emission setups with industrial and biological applications. The result showed that the system could successfully work in reasonable spatial and spectral resolution.

\section{ACKNOWLEDGMENTS}

The authors are grateful for the valuable assistance offered by Ms. Lina Beltrán Bernal and Mr. Hervé Nguendon Kenhagho during the presentation of the work. Moreover, authors would like to particularly thank Mr. Shahriar Mirpour for providing atmospheric pressure plasma jet and Mr. Mohammadreza Nazeri for his notable helps in LabVIEW code writing.

\section{REFERENCES}

[1] Garini, Y., Young, I.T. and McNamara, G., "Spectral imaging: principles and applications," Cytometry Part A, 69(8), 735-747 (2006).

[2] Li, Q., He, X., Wang, Y., Liu, H., Xu, D. and Guo, F., "Review of spectral imaging technology in biomedical engineering: achievements and challenges," Journal of biomedical optics, 18(10), 100901 (2013).

[3] Yarbrough, S., Caudill, T.R., Kouba, E.T., Osweiler, V., Arnold, J., Quarles, R., Russell, J., Otten, L.J., Al Jones, B., Edwards, A. and Lane, J., "MightySat II.1 hyperspectral imager: summary of on-orbit performance," Proc. SPIE 4480, 186-198 (2002).

[4] Gao, L., Kester, R.T., Hagen, N. and Tkaczyk, T.S., "Snapshot image mapping spectrometer (IMS) with high sampling density for hyperspectral microscopy," Optics express, 18(14), 14330-14344 (2010).

[5] Kruse, F.A., "Mapping surface mineralogy using imaging spectrometry," Geomorphology, 137(1), 41-56 (2012).

[6] Grahn, H. and Geladi, P., [Techniques and applications of hyperspectral image analysis], John Wiley \& Sons (2007).

[7] Hardeberg, J.Y., Schmitt, F.J. and Brettel, H., "Multispectral color image capture using a liquid crystal tunable filter," Optical engineering, 41(10), 2532-2549 (2002).

[8] Chang, C.I. and Du, Q., "Estimation of number of spectrally distinct signal sources in hyperspectral imagery," IEEE Transactions on geoscience and remote sensing, 42(3), 608-619 (2004).

[9] Klein, M.E., Aalderink, B.J., Padoan, R., De Bruin, G. and Steemers, T.A., "Quantitative hyperspectral reflectance imaging," Sensors, 8(9), 5576-5618 (2008).

[10] Lawrence, K.C., Park, B., Windham, W.R. and Mao, C., "Calibration of a pushbroom hyperspectral imaging system for agricultural inspection," Transactions of the ASAE, 46(2), 513 (2003).

[11] Varshney, P.K. and Arora, M.K., "Advanced image processing techniques for remotely sensed hyperspectral data," Springer Science \& Business Media (2004).

[12] Weng, Q., [Taylor \& Francis Series in Remote Sensing Applications], CRC Press, Taylor \& Francis Group (2007).

[13] Green, R.O., "Spectral calibration requirement for Earth-looking imaging spectrometers in the solar-reflected spectrum," Applied optics, 37(4), 683-690 (1998).

[14] Wu, D. and Sun, D.W., "Advanced applications of hyperspectral imaging technology for food quality and safety analysis and assessment: A review-Part I: Fundamentals," Innovative Food Science and Emerging Technologies, 19, 1-14 (2013).

[15] Nicolai, B. M., Beullens, K., Bobelyn, E., Peirs, A., Saeys, W., Theron, K. I., and Lammertyn, J. "Nondestructive measurement of fruit and vegetable quality by means of NIR spectroscopy: A review," Postharvest Biology and Technology, 46(2), 99-118 (2007).

[16] Li, Q., Xu, D., He, X., Wang, Y., Chen, Z., Liu, H., Xu, Q. and Guo, F., "AOTF based molecular hyperspectral imaging system and its applications on nerve morphometry," Applied optics, 52(17), 3891-3901 (2013).

[17] Carlomagno, G., Capozzo, L., Attolico, G. and Distante, A., "Non-destructive grading of peaches by nearinfrared spectrometry," Infrared Physics and Technology, 46(1-2), 23-29 (2004).

[18] Park, B. and Lu, R., [Hyperspectral imaging technology in food and agriculture], Springer (2015).

[19] Chen, P. and Sun, Z., "A review of non-destructive methods for quality evaluation and sorting of agricultural products," Journal of Agricultural Engineering Research, 49, 85-98 (1991). 
[20] Abbasi, H., Nazeri, M., Mireei, S.A. and Balooch, A., "LIF spectroscopy of fruits: study of excitation wavelength independence," The third Iranian Conference on Engineering Electromagnetic (ICEEM), 1-4 (2014).

[21] Abbasi, H., Bani, M.A., Nazeri, M. and Mireei, S.A., "Application of Fluorescence Spectroscopy in the Freshness Control of Lettuce Leaf after Irradiation of Cold Atmospheric Pressure Plasma," The 2nd Conference on Plasma Engineering and Physics (PEP 2014), Babolsar, Iran, 1 (2014).

[22] Noh, H.K. and Lu, R., "Hyperspectral laser-induced fluorescence imaging for assessing apple fruit quality," Postharvest Biology and Technology, 43(2), 193-201 (2007).

[23] Abbasi, H., Nazeri, M., Mirpour, S. and Farahani, N.J., "Measuring electron density, electric field and temperature of a micro-discharge air plasma jet using optical emission spectroscopy," 2nd IEEE International Conference on Knowledge-Based Engineering and Innovation (KBEI), 1139-1141(2015).

[24] Nazeri, M., Majd, A.E., Massudi, R., Tavassoli, S.H., Mesbahinia, A. and Abbasi, H., "Laser-Induced Breakdown Spectroscopy Via the Spatially Resolved Technique Using Non-Gated Detector," Journal of Russian Laser Research, 37(2), 164-171 (2016).

[25] Wang, L. and Zhao, C., [Hyperspectral Image Processing], Springer (2016).

[26] Chang, C.I., [Hyperspectral imaging: techniques for spectral detection and classification (Vol. 1)], Springer Science \& Business Media (2003).

[27] Chen, Y.R., Chao, K. and Kim, M.S., "Machine vision technology for agricultural applications," Computers and electronics in Agriculture, 36(2-3), 173-191 (2002).

[28] Abdullah, M.Z., Guan, L.C., Lim, K.C. and Karim, A.A., "The applications of computer vision system and tomographic radar imaging for assessing physical properties of food," Journal of food engineering, 61(1), 125135 (2004).

[29] Yocky, D.A., "Image merging and data fusion by means of the discrete two-dimensional wavelet transform," JOSA A, 12(9), 1834-1841 (1995).

[30] Abbasi, H., Nazeri, M. and Mireei, S.A., "Design and development of a LabVIEW-based LED-induced fluorescence spectroscopy system with applications in non-destructive quality assessment of agricultural products," Journal of Physics: Conference Series 672(1), 012010 (2016).

[31] Demtröder, W., [Laser spectroscopy: basic concepts and instrumentation], Springer Science \& Business Media (2013).

[32] Sansonetti, J.E. and Martin, W.C., [Handbook of basic atomic spectroscopic data], Journal of Physical and Chemical Reference Data, 34(4), 1559-2259 (2005). 